\title{
Structure et Fonctionnement des Ecosystèmes du Haut-Rhône Français; IX: Analyse des peuplements de deux stations phréatiques alimentant des bras morts $\left(^{*}\right)$
}

\author{
J. Gibert, R. Ginet, J. Mathieu, \\ et J.L. Reygrobellet(**)
}

\begin{abstract}
SUMMARY
Two phreatic stations providing old meanders of the French river Rhône ("Lônes") with interstitial water have been studied for three years. The samples (100 liters of water) have been collected by the Bou-Rouch method at $60 \mathrm{~cm}$ deep in the sediment.

The analyses of populations show that the biocenoses of the two stations are quite different: The one (Station 2) is rather specialized; most of the species are troglobitic ( $80 \%$ of the whole biomass; the only Amphipod, Niphargopsis casparyi, represents $67 \%$ of this biomass). The other (Station 8) is very diversified; the seven dominant groups are all epigean animals.

Population numbers have changed during the three years of sampling. Fluctuations have been observed in station 2, but the total numbers were quite similar in 1975 and 1977 . On the contrary, station 8 shows an "exponential" type of growth generated by epigean organisms, while troglobitic species remained unchanged.
\end{abstract}

A la suite d'une étude préliminaire (Gibert et al., 1977) sur le peuplement des eaux interstitielles de la Région Lyonnaise, les phénomènes biologiques liés à l'alimentation des bras morts (ou "Lônes") du Haut-Rhône par de l'eau phréatique, nous sont apparus comme les plus importants à approfondir. Pour cela, nous avons suivi pendant trois ans l'évolution des peuplements de deux stations (station 2 et 8 , in Gibert et al., 1977) alimentant des lônes dont la formation n'est pas contemporaine et dont les caractéristiques hydrologiques diffèrent sensiblement. La présentation détaillée de ces deux stations (diagrammes de circulations des eaux souterraines) est développée dans Reygrobellet et al. (in this volume).

Rappelons simplement que l'une (station 2) est située sur la rive gauche, au niveau du retour à l'état superficiel du sous-écoulement du Rhône; ceci donne naissance à un affluent d'origine interstitielle circulant parallèlement au fleuve sur plus de deux kilomètres, avant de confluer avec lui légèrement en amont du barrage de Jons (Juget et al., 1976, 1979). L'autre (station 8) se trouve, sur la rive droite du fleuve, dans la zone d'alimentation d'une lône de

(*) Publication faisant suite à l'étude hydrologique et physico-chimique des deux stations (Reygrobellet et al. 1981, dans ce volume).

${ }^{(* *)}$ Laboratoire de Biologie Souterraine, Université Claude-Bernard - LYON I, 43 Bd du 11 Novembre 1918, 69622 - VILLEURBANNE. 
formation plus récente, qui circule dans l'ancien emplacement du confluent de l'Ain avec le Rhône (carte in Gibert et al., 1977). Cette lône tire la majeure partie de son eau à la fois du sous-écoulement de l'Ain et de l'aquifère descendant du plateau de la Dombes.

\section{MATÉRIEL UTILISÉ ET MÉTHODE DE TRAVAIL}

Le matériel employé est le système de pompage "Bou-Rouch" (Bou et Rouch, 1967; Bou, 1974). Les prélèvements bimestriels, de 100 litres d'eau, s'effectuent à environ $60 \mathrm{~cm}$ sous la surface du sédiment. Etant donné le volume d'eau puisée, nous ne ferons pas référence aux classifications établies par Husmann (1974) ou Ronneberger (1975); pour notre part, nous estimons avoir, en moyenne, échantillonné entre 20 et $100 \mathrm{~cm}$ de profondeur.

La période d'échantillonnage va de début juin 1975 à janvier 1978. Elle recouvre des épisodes hydrologiques très différents, dont les conséquences physico-chimiques ont été mentionnées précédemment (Reygrobellet et al., in this volume). Il est intéressant de voir si leur impact a aussi été ressenti au niveau faunistique.

\section{RÉSULTATS}

\section{Structure générale des Biocénoses}

Pour les 3 ans de prélèvement, et pour chaque grand groupe systématique (1) des stations 2 et 8 , nous avons représenté (fig. 1):

- d'une part, l'abondance relative (A),

- d'autre part, la biomasse relative (B), calculée par l'intermédiaire des "volumes moyens." Cette valeur est déterminée de la façon suivante: dans chacun des groupes systématiques, un échantillon d'individus de tailles différentes est mesuré sous la loupe binoculaire; si l'on assimile la morphologie des individus à une forme géométrique (cylindre, cube, parallélipipède selon le cas), on peut ainsi définir le volume approximatif, puis la biomasse de l'échantillon, en considérant que la masse volumique des animaux aquatiques est voisine de l'unité.

Cette façon de procéder permet d'avoir une meilleure idée de l'influence écologique de ces groupes dans la biocénose, l'utilisation de la seule abondance relative pouvant masquer l'importance de certains organismes à biomasse forte (Renaud-Debyser, 1963; Williams et Hynes, 1974).

Il existe une trentaine de groupes systématiques dans chaque station, mais les 2 peuplements sont sensiblement différents (fig. 1):

- la station 8 possède 8 groupements principaux (2) aussi bien en abon-

(1) Les groupes systématiques ne sont pas réellement équivalents; ils représentent plus une entité écologique qu'un niveau de détermination.

(2) Les abondances relatives des autres groupements sont inférieures à $2 \%$. 

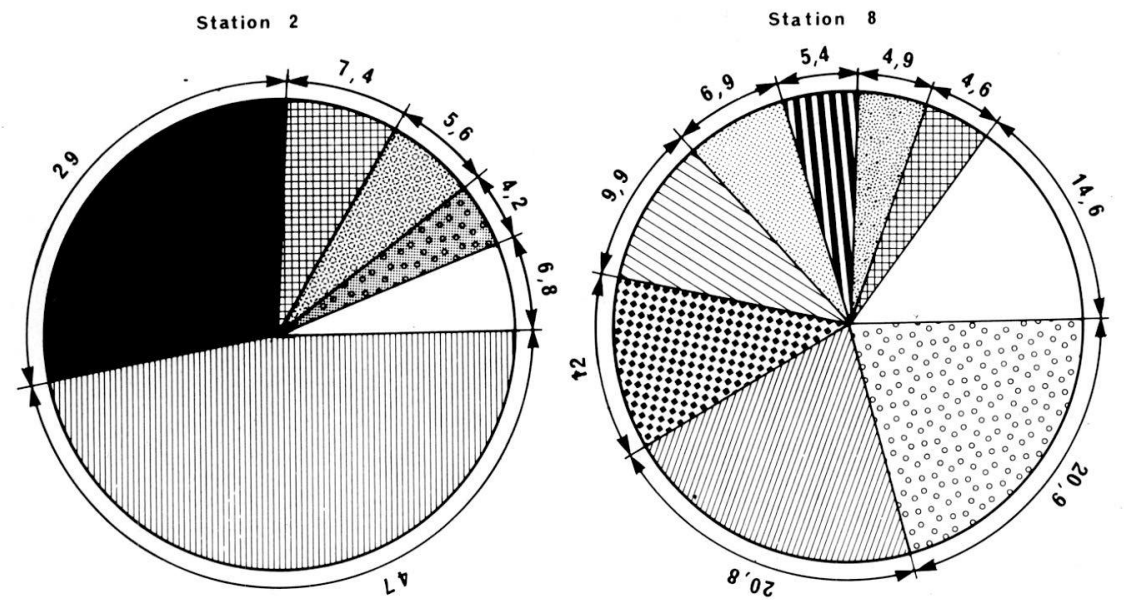

A
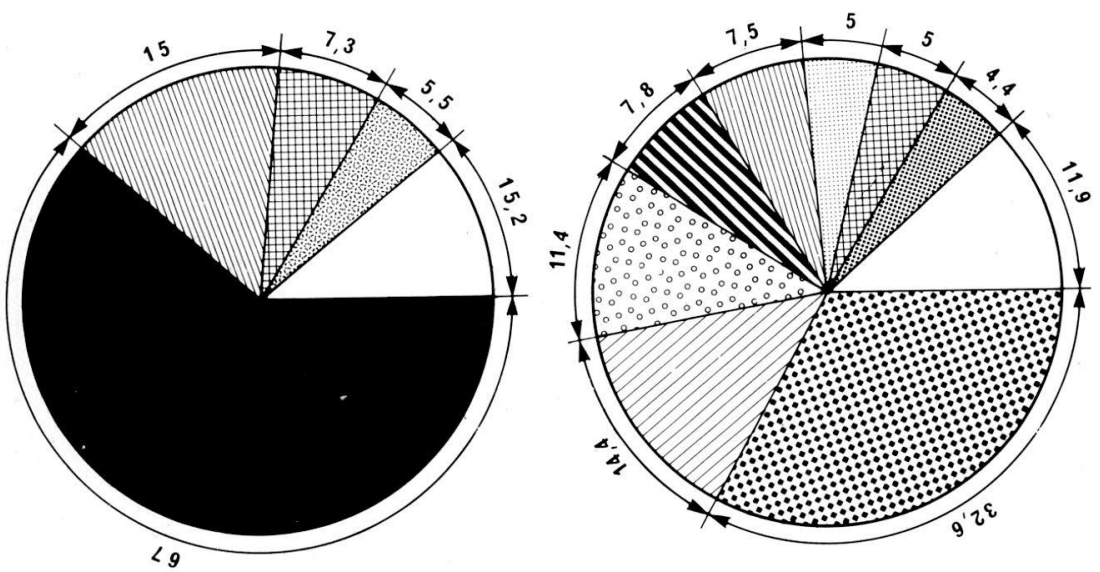

B
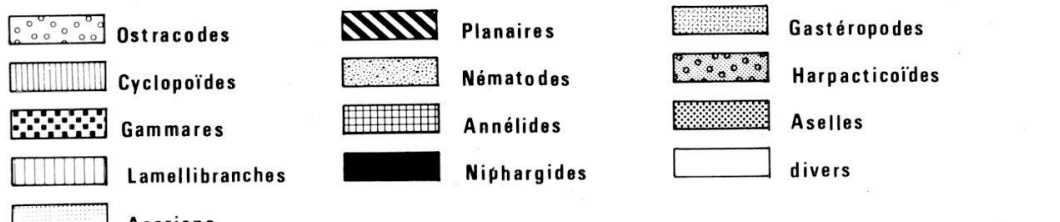

Annélides

Niphargides

divers

Fig. 1. Structure générale des biocénoses des stations 2 et 8 A: abondance relative

B: biomasse relative approchée 
dance qu'en biomasse relatives (dans le premier cas 6 sont dominants, 7 dans le second); l'expression en biomasse (B) fait rétrograder Cyclopoïdes et Ostracodes; les Nématodes disparaissent même au profit des Aselles.

- la station 2, au contraire, n'a que 5 groupements principaux (dont 4 sont dominants pour les deux types de calcul). Deux de ces groupements représentent à eux seuls $80 \%$ de toute la biocénose: les "Niphargides" (3) nettement dominés par les Cyclopoïdes lorsque leur nombre est exprimé en abondance relative, prennent toute leur signification dans le peuplement si leur pourcentage est exprimé en biomasse (67\%).

\section{Évolution des Biocénoses}

Nous avons porté sur un histogramme (fig. 2) les abondances relatives des groupes principaux cités au premier paragraphe, pour chacune des trois années de prélèvements.

Là encore, de grosses différences apparaissent:

- en station 2, les rangs de ces groupes sont assez réguliers, mais on note une baisse importante du nombre total d'individus en 1976, due à une forte diminution des Cyclopoïdes. Ce n'est pas le cas des Niphargides qui sont alors le groupe le mieux représenté. En 1977, après la grande crue de février, le peuplement retrouve sa valeur globale de 1975, mais les Annélides et les Harpacticoïdes ont augmenté, alors que les Niphargides n'ont plus que la moitié de leur effectif des deux années antérieures.

- la station 8 , très peu peuplée au début des prélèvements (un millier d'organismes seulement, où larves de Diptères et Gammares l'emportent) voit son peuplement quintupler en 1976, avec inversion des rangs. En 1977, le nombre total passe à 22.000 individus; cette progression régulière et "l'explosion démographique" de la dernière année seront analysées plus loin à un niveau systématique plus élevé.

\section{Liste Faunistique}

Nous joignons en Annexe de ce texte une liste systématique pour chacune des stations, dans l'état où se trouvent actuellement nos déterminations. Les niveaux de détermination ne sont pas tous équivalents, mais les groupes les plus abondants sont, pour la plupart, déterminés jusqu'à l'espèce; les populations en sont donc comparables. Cette liste indique, outre la "présence/absence" des unités systématiques, une estimation de leur représentation au sein du peuplement (celle-ci est exprimée en 4 classes qui tiennent à la fois compte de la fréquence et de la constance dans les prélèvements).

Un certain nombre d'informations importantes peuvent être dégagées: à ce niveau de détermination, les nombres d'unités systématiques présentes

(3) Ce terme, créé pour améliorer la rapidité de nos tris, regroupe en fait les deux genres Niphargus et Niphargopsis; la population de Niphargopsis casparyi correspond à $99,6 \%$ de ce groupe. 
dans les deux stations sont toujours très voisins (station 2: 115; station 8: 120). Cependant, pour la plupart des grands groupes (Nématodes, Oligochètes, Gastéropodes, Lamellibranches, Ostracodes, Copépodes, Amphipodes, ...), il y a très peu d'affinités entre les deux peuplements; deux exemples précis l'illustrent:
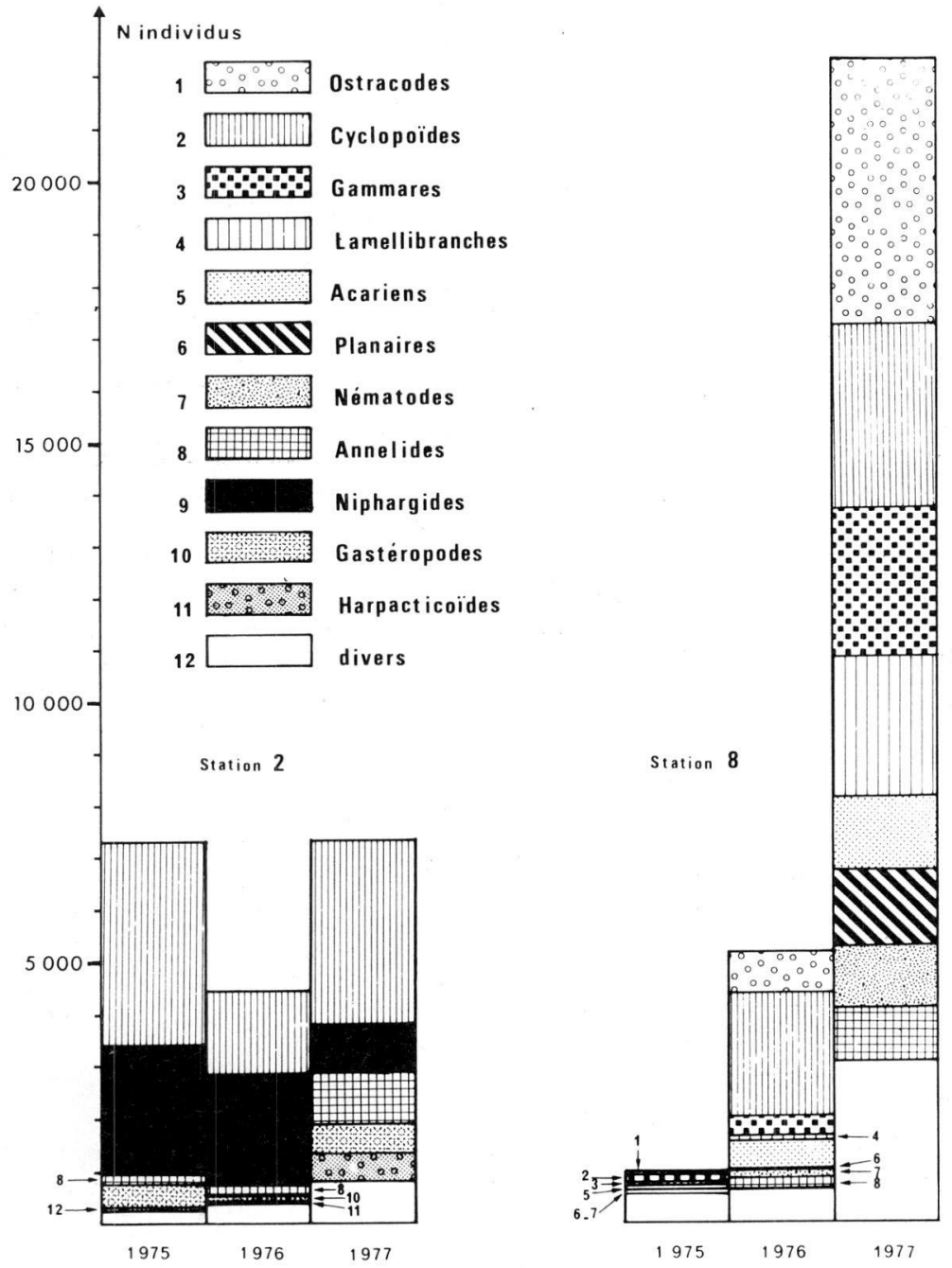

Fig. 2. Evolution de la présence des grands groupes faunistiques dans les stations 2 et 8 au cours des trois années de prélèvements. 
(a) Les troglobies

La station 2 en possède 17 unités systématiques; la station 8, 14: quoique légèrement plus élevés en station 2 , les nombres ne sont pas sensiblement différents. Cependant, ce sont les Gammaridae qui constituent pratiquement à eux seuls le nombre des troglobies de la station 8 , alors que la station 2 héberge beaucoup de très petites espèces phréatobies et troglobies, contre deux représentants seulement de la macrofaune (Niphargopsis et Niphargus).

(b) Les Oligochètes

On note un nombre d'espèces plus important en station 8 qu'en station 2 (34 contre 22). Peu d'espèces sont communes à ces deux stations ( 7 pour 46 déterminées); beaucoup sont banales et relativement bien représentées en station 8. En station 2 par contre, on remarque l'abondance de Trichodrilus leruthi, connu jusqu'à présent par quelques exemplaires récoltés dans un puits de Belgique (J. Juget, communication personnelle). On rencontre également d'autres espèces interstitielles, comme Marionina argentea ou Pristina foreli. Ces organismes étant essentiellement limivores, il est possible de relier les différences observées entre ces biocénoses avec celles de la granulométrie des deux stations; la plus forte concentration de particules assez fines (pic à 0,18 $\mathrm{mm}$ ) à la station 8 peut en effet jouer un rôle favorisant la diversité du peuplement de ces Oligochètes.

\section{Évolution au cours du temps de populations particulièrement bien représentées}

Nous avons observé des évolutions numériques différentes dans les deux peuplements (paragraphe B) pendant les trois ans de prélèvements, l'une fluctuante (2) et l'autre de type "exponentiel" (8). Les niveaux de déterminations étant insuffisants sur les diagrammes pour en suivre les détails, nous avons étudié les variations de quelques populations très abondantes dans chacune des stations:

(a) Niphargopsis casparyi

Cette espèce, connue depuis peu dans la Région Lyonnaise (Gibert et al., 1977), occupe à elle seule $67 \%$ de la biomasse totale de la station 2; les fluctuations de la population sont présentées figure 3.

Pour les deux premières années, les effectifs les plus importants sont en été (juillet/septembre), et semblent être relativement synchrones avec les maximums de température (4).

En février 77, la crue du Rhône balaye tọute la lône pendant trois semaines; elle est suivie d'un fort débit interstitiel qui se maintient pendant toute cette année très pluvieuse. Le premier prélèvement possible après la crue $(15 / 3 / 77)$ révèle la diminution brutale de la population (18 individus seule-

(4) Il faut signaler que ce phénomène s'est produit de la même façon pendant la grande sècheresse estivale de 1976, au cours de laquelle la station a été envahie par les eaux de surface, très riches en sulfates, refluant depuis le fleuve vers l'amont de la lône (Reygrobellet et al., in this volume). 


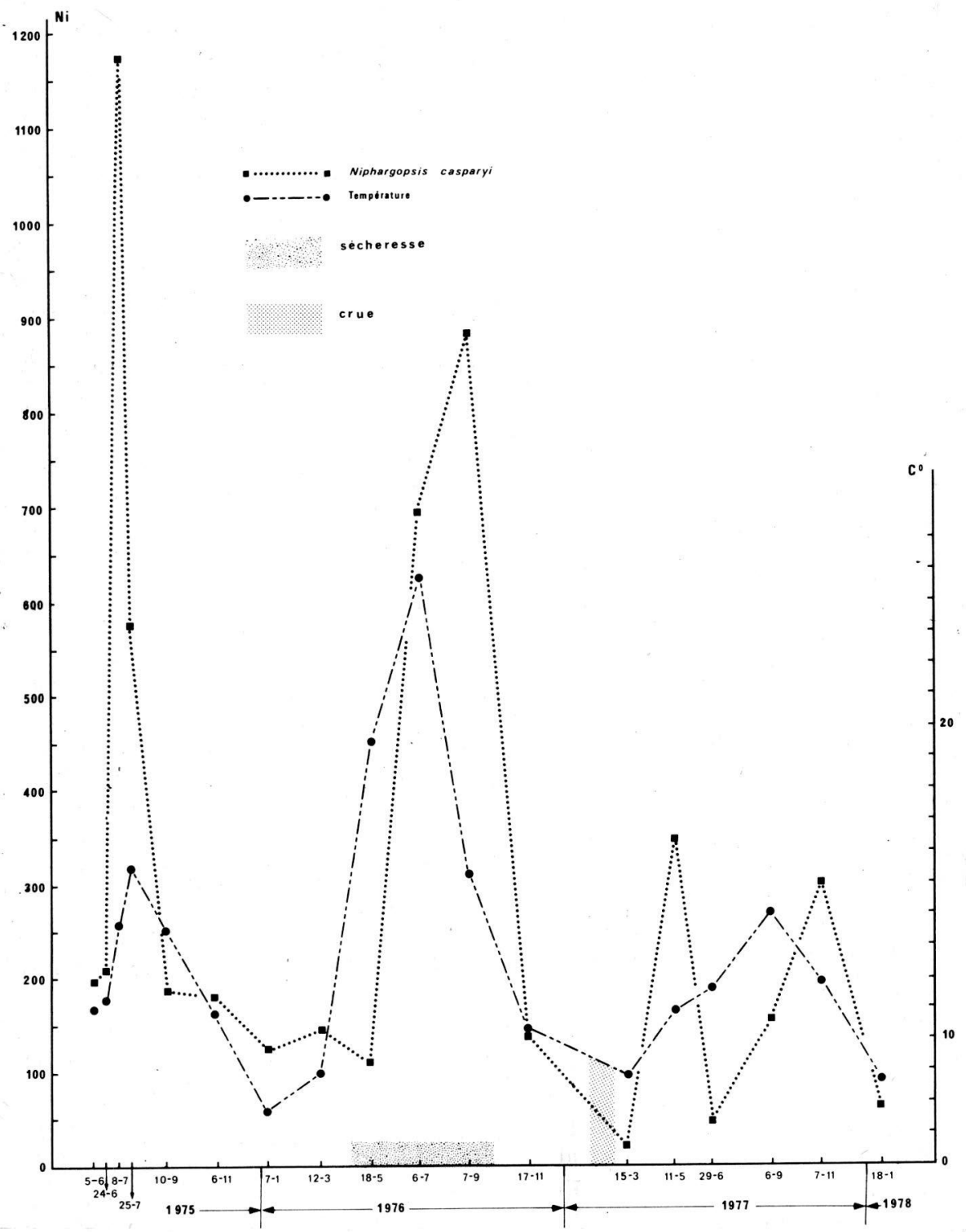

Fig. 3. Evolution de la population de Niphargopsis casparyi, et rappel de la température de l'eau correspondant à chaque prélèvement (station 2).

$\mathrm{Ni}$ : nombre d'individus présents dans un prélèvement de 1001 d'eau. 
ment pour 1001 . d'eau), elle-même liée à un appauvrissement du peuplement global (256 organismes pour 100 1.). Par la suite, deux maximums nettement inférieurs aux précédents, sont observés sur la courbe; l'un au printemps, l'autre en automne: en 1977, la population de Niphargopsis n'a donc pas retrouvé un effectif comparable à celui des deux années précédentes, alors que pendant ce délai les Cyclopoïdes, composés essentiellement de Diacyclops languidoides s.l. et Diacyclops languidus, retrouvent une densité voisine de celle de 1975.

L'effet de la crue a donc été important sur $N$. casparyi.

(b) Espèces épigées de la station 8

Les effectifs de chacun des grands groupes systématiques (fig. 2) augmentent considérablement de 1975 à 1977. sont:

Les organismes épigés principalement responsables de cette évolution

- Polycelis felina (Turbellariés)

- Pisidium sp. (Lamellibranches)

- Isohypsibius sp. (Tardigrades)

- Gammarus pulex (Crust. Amphipodes)

- Asellus aquaticus (Crust. Isopodes)

Les résultats sont représentés sur la figure 4.

Ils montrent l'importante augmentation numérique de ces cinq espèces après la crue de février 77. En revanche, le nombre des Amphipodes troglobies (représentés par 10 espèces) reste stable. Un phénomène semblable s'est produit pour les populations de Nématodes, Oligochètes, Hydracariens, Ostracodes et Cyclopoïdes, qui ont tous augmenté dans les mêmes proportions.

Cet enrichissement global de la station n'a pas totalement modifié le caractère mixte de son peuplement, où se côtoient toujours troglobies et épigés; mais à la fin de 1977, les proportions entre ces deux catégories écologiques sont devenues telles qu'on peut parler d'une très forte "superficialisation" de la communauté installée dans la station 8 . La figure 5, qui représente l'évolution du pourcentage de la biomasse troglobie par rapport à la biomasse totale de la biocénose pendant les trois ans, illustre bien ce fait.

\section{COMMENTAIRES}

Les résultats obtenus par d'autres auteurs sur différents systèmes hydrologiques sont difficilement comparables entre eux. Il existe en effet de nombreuses méthodes de prélèvement (Ronneberger, 1975); de plus, pour une même méthode (pompage), les quantités d'eau prélevées ne sont pas équivalentes: grandes quantités dans le cas présent (100 litres), pompage fractionné de faibles volumes pour DanielopoL (1976) et Gledhill (1977).

En outre, les travaux publiés montrent une diversité considérable des groupes dominants dans les différents biotopes étudiés; par exemple, en abondance relative: 
- les nappes fluviales des alluvions récentes du Tarn (France) renferment un peuplement particulièrement abondant en Amphipodes et en Copépodes (Bou, 1968). La macrofaune est bien représentée par l'Isopode Proasellus slavus dans les sédiments de la rivière yougoslave Drava (Mestrov, 1974; Mestrov et al., 1976).

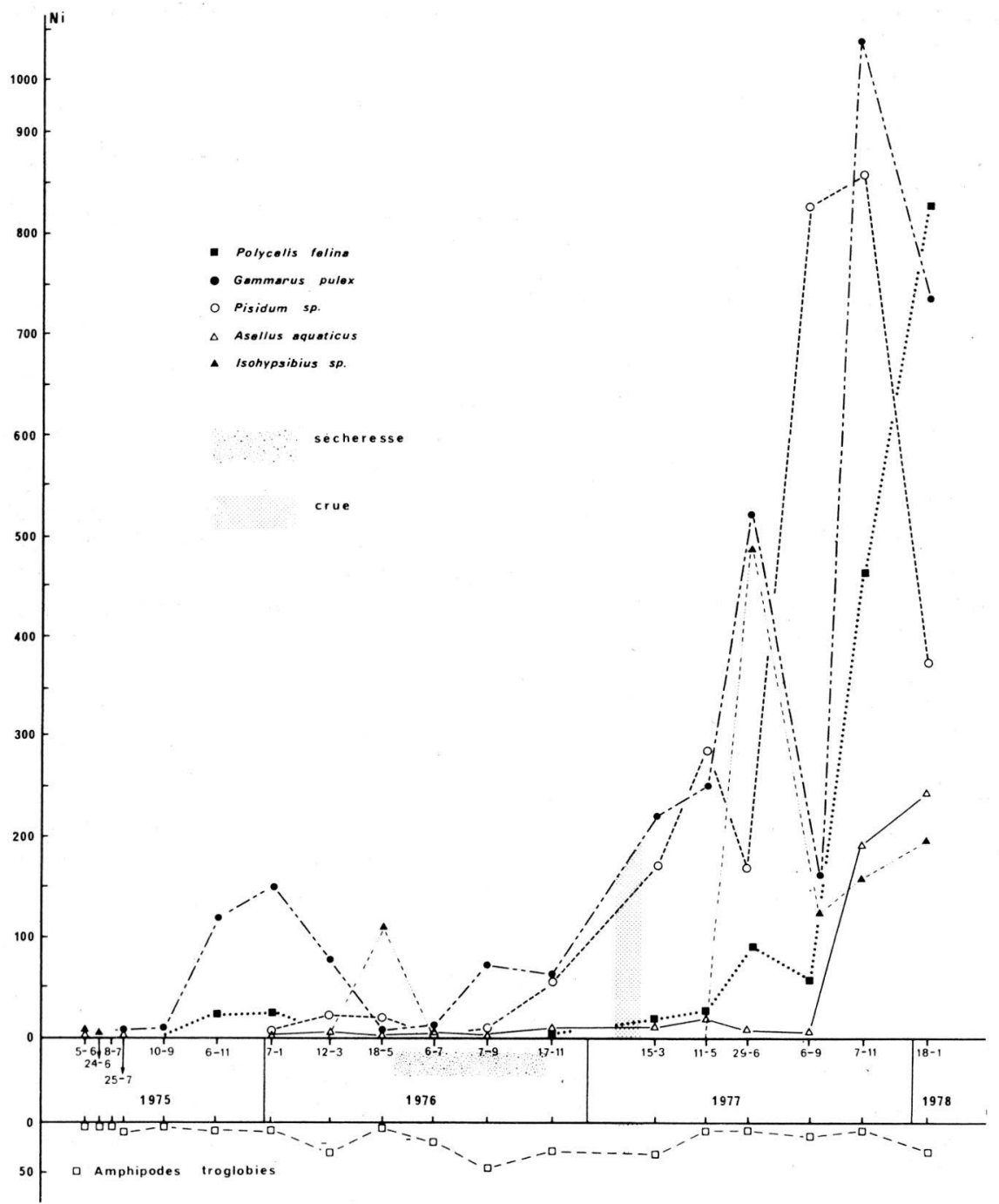

Fig. 4. Evolutions comparées de quelques populations superficielles (en haut), et du groupe des Amphipodes troglobies (en bas), a la station 8.

$\mathrm{Ni}$ : nombre d'individus présents, dans un prélèvement de 1001 d'eau. 
- par contre, les Nématodes, Oligochètes et Cyclopoïdes occupent jusqu'à $80 \%$ de la faune interstitielle du Danube autrichien (Danielopol, 1976), et sont trouvés en grande quantité en Italie dans les alluvions de l'Adige (Ferrarese et Sambugar, 1976). Dans le Breg (affluent allemand du Danube), ce sont cette fois les Harpacticoïdes qui représentent jusqu'à $50 \%$ de la faune totale (Schwoerbel, 1961).

Cependant, l'ensemble des travaux effectués ces dernières années a montré que la plupart des animaux interstitiels se concentrent dans les couches superficielles (40-60 cm de profondeur) de l'eau souterraine (Schwoerbel, 1961; Husmann, 1974, 1976; Danielopol, 1976; Mestrov et al., 1976; Bou, 1968, 1977; Williams et Hynes, 1974). C'est pourquoi nous pensons que la méthode employée ici (pompages de grands volumes dans la zone la plus peuplée), si elle ne nous renseigne pas sur la distribution verticale des organismes dans le sédiment, nous permet une bonne estimation des biocénoses en place.

Nous ne confronterons donc pas longuement nos résultats quantitatifs avec d'autres; ils montrent en effet que, chacune à leur manière, les stations 2 et 8 sont différentes de celles des sédiments alluvionnaires fluviaux déjà connus. Nous énumèrerons simplement les principales informations que les paragraphes précédents ont apportées sur les biocénoses qui y résident.

(a) Considérée globalement sur l'ensemble des prélèvements, la station 2 (dont le pourcentage de troglobies et phréatobies peut être estimé à $50 \%$ en abondance relative et à $80 \%$ en biomasse approchée) apparaît comme nettement plus "hypogée" que la station 8 (où les espèces souterraines représen-

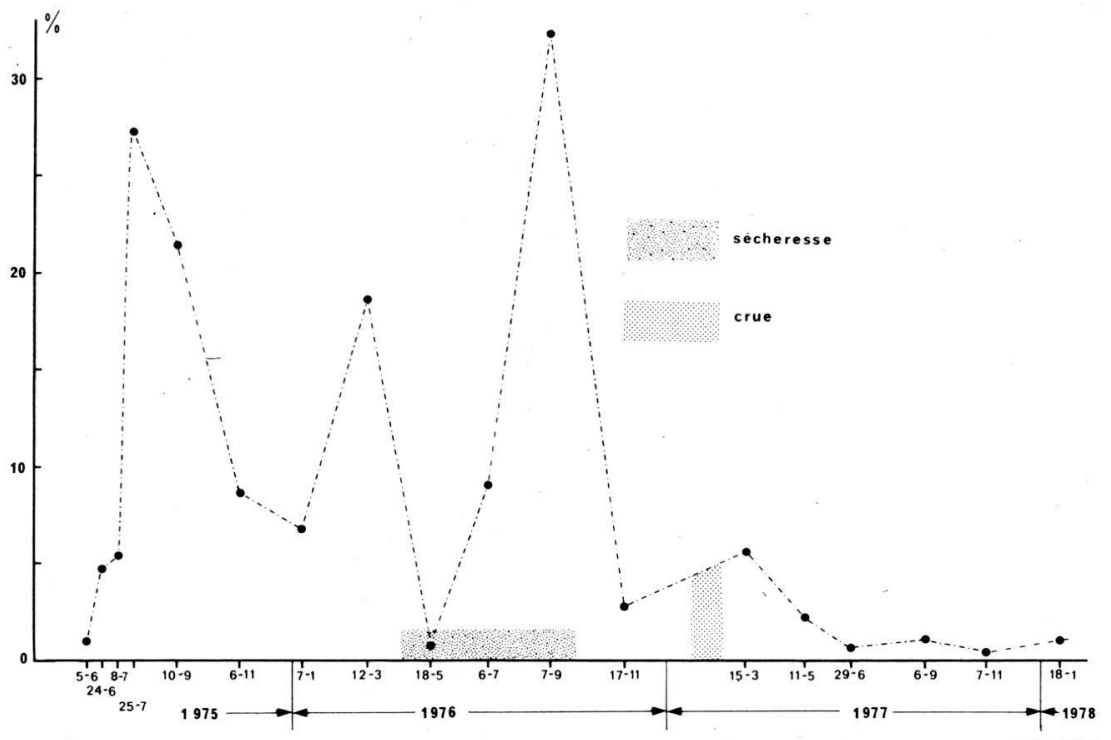

Fig. 5. Estimation du pourcentage de la biomasse des troglobies par rapport à la biomasse totale de la biocénose, pour chaque prélèvement, pendant trois ans (station 8). 
tent $8,2 \%$ en biomasse, et seulement $1 \%$ en abondance relative). Elle est également la plus spécialisée des deux stations, Niphargopsis casparyi étant très fortement dominant dans le peuplement.

(b) la station 8 présente, d'une façon générale, une très forte concentration de populations de type épigé, qui pénètrent jusqu'à au moins un mètre sous la surface du sédiment. Elle apparaît très riche et diversifiée. Les nombres d'individus sont comparables à ceux cités par Williams et Hynes (1974) pour une profondeur voisine. L'importance écologique de cette "couche biologique superficielle souterraine" (Gibert et al., 1977) n'est plus à démontrer, pas plus que son éventuelle vulnérabilité aux agressions du milieu extérieur (Mestrov, 1974; Mestrov et al., 1976; Bou, 1977; Danielopol, 1976).

(c) Les variations quantitatives observées dans les deux peuplements sont en apparente opposition avec les caractéristiques hydrologiques des stations: en station 2 , à un régime très variable étroitement lié à celui du Rhône vif, correspond un peuplement qui a assez peu évolué pendant la durée de notre étude. Dans le même temps, celui de la station 8 , qui semblait devoir être à priori le plus stable (l'eau alimentant cette station est très tamponnée par l'apport considérable de l'aquifère issu de la plaine de La Valbonne), a une croissance de type exponentiel.

(d) Ce phénomène d'explosion démographique, observé depuis le printemps 1977 en station 8, peut être interprété de plusieurs façons différentes:

- Le brusque apport de limon et de matière organique après la grande crue du Rhône est susceptible d'avoir modifié le milieu à un point tel que des organismes épigés, amenés de l'amont, ont pu se maintenir et se multiplier dans le substrat; en même temps, les populations locales ont pu se développer dans un contexte rendu plus favorable, déterminant ainsi ce peuplement très riche dont la stabilité à moyen terme n'est pas assurée. Signalons cependant qu'en mars 1978 (résultats non représentés sur les figures 4 et 5), celui-ci continue d'augmenter. Williams et Hynes (1974), et avant eux Schwoerbel (1961) puis Egglishaw (1964) ont constaté des faits très semblables, Williams et Hynes allant même jusqu'à créer les termes de "occasional and permanent hyporheos."

- A contrario, le pompage régulier à un endroit précis d'une grande quantité d'eau peut avoir déblayé le limon fin et fait naître de nouveaux interstices au sein du sédiment; ils auraient ainsi servi de lieu de refuge ou de concentration pour la faune. Mais, nos prélèvements étant effectués tous les deux mois dans ce courant de rééquilibrage (dont le débit est toujours suffisant pour mettre en suspension dans la lône des sables grossiers), il nous semble que le recolmatage des vides créés artificiellement par le pompage ne devrait pas demander autant de temps. Rappelons, de plus, que rien de comparable n'a été observé en station 2, ni dans d'autres stations dont nous nous occupons actuellement.

- Les courants de rééquilibrage du niveau piézométrique évoluent dans le temps; peut-être celui de la station 8 s'est-il renforcé naturellement, engendrant pour certaines espèces rhéophiles un "ffet de source" (Gammarus pulex, Polycelis felina)? 
Dans l'état actuel de nos connaissances, il semble qu'aucune de ces trois hypothèses ne puisse être écartée a priori.

Les résultats commentés ci-dessus ne concernent qu'une analyse globale des variations observées sur ces populations interstitielles. Par la méthode employée ici, les zonations verticales et les diverses migrations animales ne peuvent être étudiées. L'implantation de substrats artificiels, assez utilisés pour l'échantillonnage du benthos (Roux et al., 1976), sert déjà à des recherches de zonation verticale (Poole et Stewart, 1976); après avoir été adaptée à la granulométrie de nos milieux interstitiels, et après la mise au point d'une technique de tri appropriée, il semble que cette méthode puisse être un test comparatif pour les pompages, et que son emploi constituerait une voie de recherche très positive pour l'avenir.

\section{RÉSUMÉ}

Deux stations phréatiques dont l'eau alimente des bras morts (Lônes) ont été étudiées simultanément pendant trois années consécutives. Les prélèvements (100 litres) ont été effectués à $60 \mathrm{~cm}$ sous la surface du sédiment par la méthode de pompage "BOU-ROUCH."

Les peuplements analysés globalement montrent que les stations abritent des biocénoses très différentes. L'une (Station 2), s'avère assez spécialisée et très nettement hypogée $-80 \%$ de sa biomasse totale est troglobie, et $67 \%$ sont représentés par le seul Amphipode Niphargopsis casparyi - . L'autre (Station 8), est très diversifiëe; ses 8 groupes principaux sont constitués par des organismes superficiels.

Les peuplements ont évolué au cours des trois années de prélèvements. Celui de la station 2 a subi des fluctuations, mais retrouve son effectif initial en 1977. Au contraire, celui de la station 8 accuse une croissance de type "exponentiel" dont les responsables sont ces groupes superficiels, les troglobies restant stables.

\section{OUVRAGES CITÉS}

BOU, C. 1968. Faune souterraine du sud-ouest du Massif-Central. II - Contribution à la connaissance de la faune des eaux souterraines de l'Albigeois. Ann. Spéléol. 3(2):29-31.

BOU, C. 1974. Recherches sur les eaux souterraines - 25 - Les méthodes de récolte dans les eaux souterraines interstitielles. Ann. Spéléol. 29(4):611-619.

BOU, C. 1977. Conséquences écologiques de l'extraction des alluvions récentes dans le cours moyen du Tarn. Bull. Ecol. 8(4):435-444.

BOU, C. et R. ROUCH. 1967. Un nouveau champ de recherches sur la faune aquatique souterraine. C.R. Acad.Sci. Paris 265:369-370.

DANIELOPOL, D.L. 1976. The distribution of the fauna in the interstitial habitats of riverine sediments of the Danube and the Piesting (Austria). Int. J. Speleol. 8:23-51.

EGGLISHAW, H.J. 1964. The distributional relationship between the bottom fauna and plant detritus in streams. J. Anim. Ecol. 33:463-476.

FERRARESE, U. et B. SAMBUGAR. 1976. Ricerche sulla fauna interstiziale iporreica dell'Adige in relazione allo stato di inquinamento del fiume. Rivista di Idrobiologia, $X V, 1: 47-124$.

GIBERT, J., R. GINET, J. MATHIEU, J.L. REYGROBELLET, et A. SEYED-REIHANI. 1977. Structure et fonctionnement des écosystèmes du Haut-Rhône français. IV - le peuplement des eaux phréatiques; premiers résultats. Ann. Limnol. 13 1:83-97.

GLEDHILL, T. 1977. Numerical fluctuations of four species of subterranean amphipods during a five year period. Crustaceana Suppl. 4:144-152.

HUSMANN, S. 1974. Versuche zur Erfassung der vertikalen Verteilung von Organismen und chemischen Substanzen im Grundwasser von Talauen und Terrassen; Methoden und erste Befunde. Int. J. Speleol. 6:271-302. 
HUSMANN, S. 1976. Langsamsandfilter als Lebensraüme von Grundwassertieren, mit Befunden aus Wiesbaden-Schierstein. Wissenschaftliche Berichte über Untersuchungen und Planungen der Stadtwerke Wiesbaden AG, Band 3:83-92.

JUGET, J., C. AMOROS, D. GAMULIN, J.L. REYGROBELLET, M. RICHARDOT, Ph. RICHOUX, et C. ROUX. 1976. Structure et fonctionnement des écosystèmes du HautRhône français. II - Étude hydrologique de quelques bras morts. Premiers résultats. Bull. Ecol. 7(4):479-492.

JUGET, J., B.J. YI, C. ROUX, Ph. RICHOUX, M. RICHARDOT-COULET, J.L. REYGROBELLET, et C. AMOROS. 1979. Structure et fonctionnement des écosystème du Haut-Rhône français. VII - Le complexe hydrographique de la Lône des Pêcheurs (ancien méandre du Rhône). Rev. Suisse Hydrol. 41/2:395-417.

MESTROV, M. 1974. Les données des recherches écologiques et faunistiques des eaux interstitielles le long des rivières Sava et Drava concernant la pollution. 29th Congr. Int. Assoc. Limnol., Canada 1974; abstracts:138.

MESTROV, M., R. LATTINGER-PENKO, et V. TAVCAR. 1976. La dynamique de population de l'Isopode Proasellus slavus ssp.n. et les larves de Chironomides dans l'hyporhéique de la Drave du point de vue de la pollution. Int. J. Speleol. 8:157-166.

POOLE, C., et W. STEWART. 1976. The vertical distribution of macrobenthos within the substratum of the Brazos river, Texas. Hydrobiologia 50 (2):151-160.

RENAUD-DEBYSER, J. 1963. Recherches écologiques sur la faune interstitielle des sables Bassin d'Arcachon, île de Bimini, Bahamas. Vie et Milieu, supplément $n^{\circ}$ 15:157 p.

$\bar{R} \bar{E} \bar{Y} \bar{G} \bar{R} O \bar{B} \bar{B}$ LLLET, J.L., J. MATHIEU, R. GINET, et J. GIBERT. 1981. Structure et fonctionnement des écosystèmes du Haut-Rhône français. VIII - Hydrologie de deux stations phréatiques dont l'eau alimente des bras morts. Int. J. Speleol. (in this volume).

RONNEBERGER, D. 1975. Zur Kenntniss der Grundwasserfauna des Saale-Einzugsgbietes (Thüringen). Limnologica, Berlin 9(3):323-419.

ROUX, A.L., H. TACHET, et M. NEYRON. 1976. Structure et fonctionnement des écosystèmes du Haut-Rhône français. III - Une technique simple et peu onéreuse pour l'étude des macroinvertébrés benthiques des grands fleuves. Bull. Ecol. 7(4):493-496.

SCHWOERBEL, J. 1961. Uber die Lebensbedingungen und die Besiedlung des hyporheischen Lebensraumes. Arch. Hydrobiol. Suppl. 25:182-214.

WILLIAMS, D.D., et N.B. HYNES. 1974. The occurrence of benthos deep in the substratum of a stream. Freshwater Biol. 4:233-256.

\section{LISTE FAUNISTIQUE}

Certaines détermination̨s ont été effectuées par des spécialistes qui ne sont pas les auteurs ou en collaboration avec eux; qu'ils veuillent bien trouver ici l'expression de nos remerciements:

Plathelminthes: E. Pattée, Nématodes et Oligochètes: J. Juget, Mollusques: R. Bernasconi et M. Coulet, Tardigrades: G. Ramazzotti, Cladocères: C. Amoros, Cyclopoïdes: F. Lescher - Moutoué, Harpacticoïdes: R. Rouch, Ostracodes: D. Danielopol, Isopodes: J.P. Henry, Trichoptères: H. Tachet, Coléoptères: P. Richoux.

Sur cette liste, chaque unité systématique, présente dans l'une ou l'autre station, est accompagnée d'une estimation de sa représentation au sein de la biocénose. Celle-ci est exprimée en 4 classes, déterminées à la fois:

- par la constance dans les prélèvements,

- et par l'abondance relative (pondérée par la biomasse approchée)

Ainsi, les unités systématiques rares sont accompagnées d'un petit carré blanc, et les autres d'un carré noir dont la surface est proportionnelle à leur importance dans le peuplement. 
CNIDAIRES

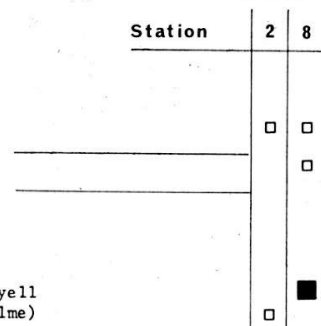

NĚ MATHE LMI NTHES

Hydrozoaires

- Hydridae ind. (1)

GORDIENS ind.

PLATHELMINTHES

Turbellariés

Planariidae

- Polycelis felina Dalyel

- Polycelis felina Dalyell
- g. sp. (anophtalme)

Nématodes (2)

- Plectidae

- Plectus cirratus (Bastian, 1865)

- Plectus rhizophilus (De Man, 1880)

- Monhisteridae

- Theristus setosus (Bütschli)

- Tripylidae

- Tripy la filicaudata

- Trichistoma monohystera

- Tobmilus grandipapiliatus

onchidae

- Mononchus trroncatus Bastian, 1865

- Mononchus papizlatus Bastian, 1865

- Paractinolaimidae

- Paractinolaims sp.

- Dorylaimidae

- Mesodomy Zaimus sp.

- Qudsianematidae

- Eudory laimus sp.

- Aporce laimidae

- Aporce laime llus sp.

- Mermi thidae

- Mermis sp.

- g. sp. ind

ANNÉLIDES

Oligochètes

- Aelosomatidae

- Aelosoma hemprichi Ehrenberg, 1828

- Aelosoma sp.

- Naididae

- Chaetogaster langi Bretscher, 1896

- Chaetogaster diastrophus (Gruithuisen, 1928)

- Amphichaeta leydigii Tauber, 1879

- Specaria josinae (Vejdovsky, 1833)

- Uncinais uncinata (Orsted, 1842)

- Ophidonais serpentina (Müller, 1773)

- Nais commoris Piguet, 1906

- Nais vamabilis Piguet, 1906

- Nais simplex Piguet, 1906

- Nais alpina Sperber, 1948

- Nais barbata Müller, 1773

- Nais pseudobtusa Piguet, 1906

- Nais pardalis Piguet, 1906

- Nais elinguis Müller, 1773

- Slavina appendiculata (D'Udekem, 1855)

- Vjedovskiella intermedia (Bretscher, 1896)

- Stylaria lacustris (Linné, 1767)

- Dero digitata (Müller, 1773)

- Dero obtusa d'Udekem, 1855

- Pristina foreli (Piguet, 1906)

- Pristina idrensis Sperber, 1948

口

(1) Vuel'extrême difficulté de détermination de ce matériel (élevage nécessaire) nous n'avons pas jugé bon de préciser au-delà. Seule, Pelmatohydra oligactis (Pallas) a puêtre identifiée dans 2 stations voisines (Dét. $x$. Kolenkine, Lyon)

(2) Pour les Nématodes, nous ne fournissons qu'une information de présence. Les résultats quantitatifs ne sont Ras connus.

$\rightarrow$ * espècesconsidérées comme phréatobies ou troglobies 
- Tubificidae

- Aulodrilus pluriseta (Piguet, 1906)

- Aulodrilus limnobius Bretscher, 1899

- Tubifex ignotus (Stolc, 1886)

- Psamoryctides barbatus (Grube, 1861)

- Potamothrix heuscheri (Bretscher, 1900)

- Potamothrix vejdovskyi (Hrabe, 1941)

- Limnodrilus hoffmeistemi Claparède, 1862

- Rhyacodrilus coccinaeus (Vejdovsky, 1875)

- Rhyacodrilus sp.

- Lumbriculificidae ind. immatures

- Stylodrilus heringionus Claparède, 1862

- Stylodrilus parvus (Hrabe et Cernosvitov, 1927)

- Trichodrilus leruthi (Hrabe, 1937)

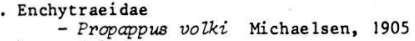

- Achaeta sp.

- Achaeta sp.

(Nielsen et Christensen, 1959)

- Cernosvitoviezla atrata

- Cognettia grandulosa (Michaelsen, 1898)

- Enchytraeus bucholzi Vejdovsky, 1879

- Marionina argentea (Michaelsen, 1899)

- Marionina riparia Bretscher, 1899

- Marionina sp.

- Haplotaxidae

- Haplotaxis gordioides Michaelsen, 1900

Achètes

- Glossiphoniidae

- Glossiphonia complanata (Linné 1758)

MOLLUSQUES

\section{Gastéropodes}

- Cochlostomidae

$$
\text { - Cochlostoma sp. (3) }
$$

- Hydrobiidae

- Hauffenia minuta minuta Draparnaud

- Valloniidae

- Vallonia pulchelza Müller, 1774 (3)

- Pupillidae

- Vertigo sp. "(3)

- Ferussaciidae

- Caecrilioides acioúla, Müller 1774 (3)

- Helicidae

- Helice Zla sp.

- Ellobidae

- Carichium tridentatum Risso, $\$ 826$

- Lymnaeidae

- Radix peregra Müller, 1774

- Planorbidae

- Arriger crista (Linné, 1758)

- Ancylidae

- Acroloxus lacustris (Linné, 1758)

Lame 11 ibranches

- Sphaeridae

- Pisidium sp.

TARDI GRADES

- Isohypsibius sp.

ARTHROPODES

\section{Arachnides}

Acariens

Prostigmates

- Hydracariens ind.

lés os tigmates

- Gamasidae ind. (4)

Oribates ind. (4)
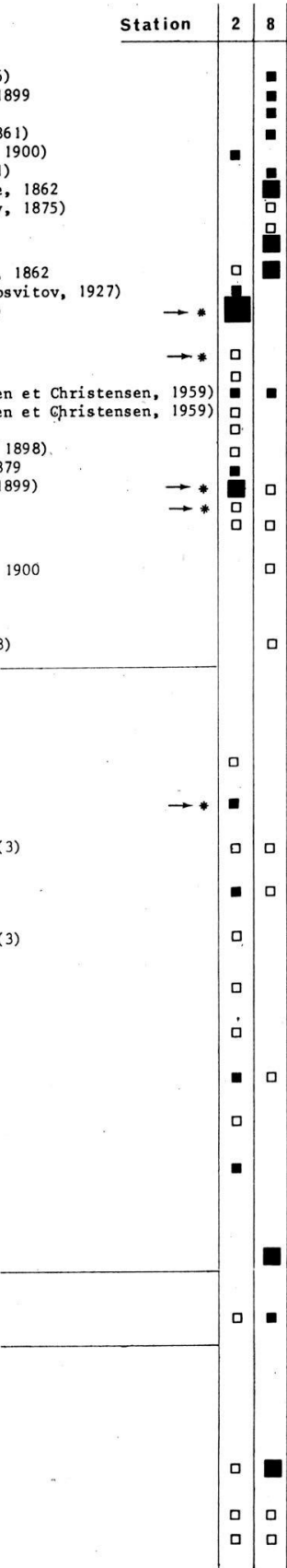

(3) espèces connues comme terrestres

(4) exogènes appartenant à la faune du sol 
Crustacés

Cladocères

- Daphniidae

- Daphnia longispina O.F. Müller, 1785

- Ceriodaphnia reticulata (Jurine, 1820)

- Ceriodaphnia megoss Sars, 1862

- Simocephalus expinosus (Koch, 1841)

- Scapholeberis mucronata (O.F. Múller, 1785)

- Bosminidae

- Bosmina (Bosmina) Zongirostris (0.F. Müller, 1785)

- Bosmina sp.

- Macrothricidae

- Iliocryptus sordidus (Liêvin, 1848)

- Chydoridae

- Evrycercus Zamellatus (0.F. Müller, 1785)

- Acroperus harpae (Baird, 1835)

- Alona guttata Sars, 1862

- Alona affinis (Leydig, 1860)

- Disparalona rostrata. (Koch, 1841)

- Pleuroxus uncinatus Baird, 1850

- Pleuroxus adioncus (Jurine, 1820)

- Chydorus sphaericus (0.F. Müller, 1785)

- Monospilus dispar Sars, 1862

Os tracodes

- Cypridae

- Candona candida (O.F. Müller, 1785)

- Candona aff. Lobipes Hartwig, 1900

- Candona aff. schellenbergi Klie, 1934

- Candona compressa

- Candona aff. Hertzogi Klie, 1934

- Candona sp.

- Pseudocardona sp.

- Cryptocandona sp.

- Cyclocypris ovum (Jurine, 1820)

- Cipria ophtalmica (Jurine, 1820)

- Eucypris aff. serrata (G.w. Müller, 1900)

- Ilyocypris sp.

- Herpe tocypris reptans (Baird, 1835)

- Cypridopsis vidua (0.F. Müller, 1776)

- Potamocypris aff. variegata (Brady et Norman, 1889)

- Potamocupris s.p.

Copépodes

Calanoïdes ind.

Cyclopoïdes

- Cyclopidae

- Macrocyclops albidus (Jurine, 1820)

- Eucyclops serrulatus (Fischer, 1851)

- Eucyciops macruroïdes (Lilljeborg, 1908)

- Tropocyclops prasinus (Fischer, 1860)

- Paracyclops fimbriatus (Fischer, 1853)

- Acanthocyclops (Acanthocyclops) robus tus (G.0. Sars, 1823)

- Acanthocyclops (Acanthocyclops) sensitivus (Graeter et Chappuis, 1914)

- Acanthocyclops (Acanthocyclops) venustus (Norman et Scott, 1906)

- Acanthocyclops (Acanthocyclops) of. troglophilus Lescher-Moutoue $\rightarrow$ *

- Acartinocyclops (Megacyclops) viridis viridis (Jurine, 1820)

- Diacyclops bicuspidatus (Claus, 1857)

- Diacyclops bisetosus (Rehberg, 1880)

- Diacyclops Zangridus (G.0. Sars, 1863)

- Diacyclops Zanguidoïdes clandestinus (Kiefer, 1926)

- Diacyclops languidoïdes putealis (Chappuis, 1928)

- Diacyclops languidoides hypnicola (Gurney)

- Diacyclops languidoïdes s.s.p.

- Graeteriella (Graeteriella) misetigera (Graeter, 1908)

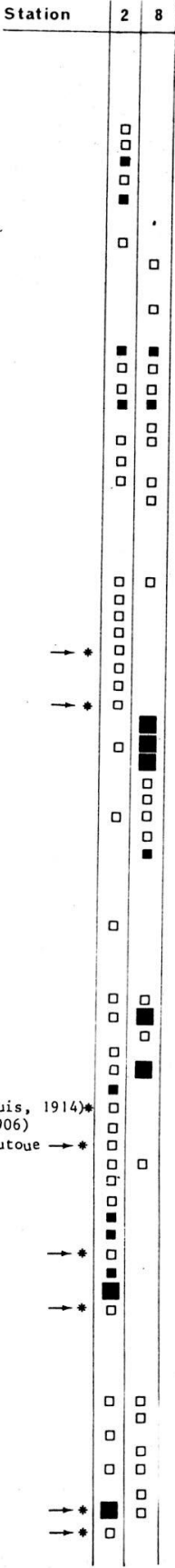

- Canthocamp tidae

- Canthocamptus staphylinus (Jurine, 1820)

- At theyeila (Attheyella) crassa (Sars, 1863)

- Attheyelza (Mrazekiella) wulmeri (De Kerhervé, 1914)

- Bryocamptus (Bryocamptus) minutus (Claus, 1863)

- Bryocamptus (Bmocamptus) zschokkei (Schmeil, 1893)

- Bryocamptus (Limocamptus) echinatus Mrazek, 1893

- Elaphoide zla e laphoides

- Parastenoscaris sp. 
Amphipodes

- Gammaridae

- Gammarus pulex (Linné , 1758)

- Gammarus fossarum Koch, 1836

- Cammarus sp. anophtalme (5)

- Niphargopsis casparyi Pratz, 1932

- Niphargus rhenorhodonensis (Sche

- Niphargus kochianus Bate, 1863

- Niphargus jovanovici Karaman, 1931

- Niphargus sp.

- Niphargus nov. sp. (6)

- Salentinella juberthiae

- Salentine ila de lamarei

- Salentine lla sp.

- Crangony $x$ subterraneus

N. Coineau, 1968

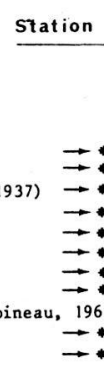

Bate, 1859

\section{Is opodes}

- Asellidae

- Asellus aquaticus Linné

- Proasellus walteri (Chappuis, 1948)

- Microparasellidae

- Microcharon sp.

Insectes

\section{Ap térygotes}

Collemboles (4)

- Sminthuridae

- Dicyrtomidae

- Entomobryidae

- Isotomidae

- Poduridae

(7)

$$
\text { Ptérygotes }
$$

\section{Plécoptères}

- Nemuridae

- Nemura sp. 1. Juv.

- Leuctridae

- Leuctra $o p$. 1. Juv.

Ephëémérogtềres

- Baetidae ind. 1. Juv.

- Caenidae

- Caeris robusta 1. Juv.

- Caenis sp. 1. Juv.

\section{Ir $\underline{\text { ich }}$ honortè res}

\section{- Sericostomatidae}

- Sericos toma sp. L.Juv.

: Goeridae

- Sizosp. 1. Juv.

Coléeortères

- Haliplidae

- Haliplus lineaticollis (Marsham 1802) 1.+ a

- Haliplus rufficollis (De Geer 1775) a.

- Dytiscidae

- Hydroporus sp.

- Dryopidae

- Dryops sp.

- Elmidae

- Esolus parallelepipedus (Müller, 1806) $1 .+$ a .

- Limius volkmari (Panzer, 1793) 1.

\section{Hệtéróptères}

- Corixidae ind. Juv.
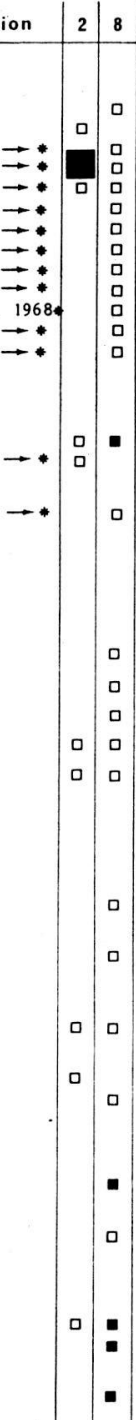

(5) appartiennent au groupe pulex

(6) espèce inconnue en cours d'examen par S. RUFro

(7) 1 . = larves

a. adultes 


\begin{tabular}{|c|c|c|}
\hline Station & 2 & 8 \\
\hline Diptères L. & & \\
\hline $\begin{array}{l}\text { Chi ronomidae } \\
\text { - Chironomiens } \\
\text { - Tanypodinae } \\
\text { - Tany tarsiens } \\
\text { - Orthocladiinae } \\
\text { - Coryneroinae } \\
\text { - Diamesinae } \\
\text { - Juvéni les inde terminés }\end{array}$ & $\begin{array}{l}0 \\
0 \\
0 \\
0\end{array}$ & $\begin{array}{l}\text { a } \\
\text { a } \\
\text { a } \\
\text { a } \\
\text { D }\end{array}$ \\
\hline Ceratopogonidae (type Bezzia) & & - \\
\hline Simulidae & & - \\
\hline Tabani dae & 0 & \\
\hline Autres diptères ind. & & 口 \\
\hline Psogcoptèreses (1). & 0 & \\
\hline
\end{tabular}

